\title{
Revisiting the anisotropy of metamaterials for water waves
}

\author{
A. Maurel, J.-J. Marigo, P. Cobelli, P. Petitjeans, and V. Pagneux \\ I. Langevin/ESPCI, 1 rue Jussieu, 75005 Paris, France; \\ LMS/Ecole Polytechnique, route de Saclay, 91120 Palaiseau, France; \\ Dpt Fisica/Univ. Buenos Aires and IFIBA, Cuidad Universitaria, Buenos Aires 1428, Argentina; \\ PMMH/ESPCI, 10 rue Vauquelin, 75005 Paris, France; \\ and LAUM/Univ. du Maine, Av. O. Messiaen, 72085 Le Mans, France
}

(Received 23 July 2017; revised manuscript received 3 October 2017; published 26 October 2017)

\begin{abstract}
We establish, both theoretically and experimentally, that metamaterials for water waves reach a much higher degree of anisotropy than the one predicted using the analogy between water waves and their electromagnetic or acoustic counterparts. This is due to the fact that this analogy, based on the two-dimensional shallow water approximation, is unable to account for the three-dimensional near field effects in the water depth. To properly capture these effects, we homogenize the fully three-dimensional problem and show that a subwavelength layered structuration of the bathymetry produces significant anisotropic parameters in the shallow water regime. Furthermore, we extend the validity of the homogenized prediction by proposing an empirical anisotropic version of the dispersion relation.
\end{abstract}

DOI: 10.1103/PhysRevB.96.134310

\section{INTRODUCTION}

The design of metamaterials for water waves has generated in the past 10 years an increasing interest for applications including the realization of lenses [1-3], the control of the ocean wave energy flow [4-6], or the cloaking able to produce a protected free wave region [7-13]. For most of the cases, these metamaterials require anisotropic effective parameters that can take extreme values, a task that can be challenging. One strategy to design artificial anisotropic media consists of using a bathymetry varying at the subwavelength scale. The waves propagating over such a structured medium feel effective water depths differently depending on its direction of propagation. Often, heuristically, the effective water depths have been evaluated using the homogenization of the twodimensional wave equation under the linear shallow water approximation (2D SWE)

$$
\operatorname{div}(h \nabla \eta)+\frac{\omega^{2}}{g} \eta=0,
$$

thus benefiting from the analogy with equations of the Helmholtz type valid for polarized electromagnetic waves and acoustic waves $[1,5,6]$. In $(1), \eta(x, y)$ is the surface elevation, $h(x, y)$ is the local water depth, $\omega$ the frequency, and $g$ the gravity constant. Note that, starting from an initially $3 \mathrm{D}$ water wave problem, the shallow water approximation allows us to reduce the effect of the third dimension $z$ in the inhomogeneous factor $h$. For structurations made of layers $[5,6]$, this modeling predicts an effective water depth tensor related to the arithmetic and geometric averages of the actual water depths; the effective tensor is diagonal with

$$
\text { 2D SWE } h_{\mathrm{x}}=\left\langle h^{-1}\right\rangle^{-1}, \quad h_{\mathrm{y}}=\langle h\rangle,
$$

in $(x, y)$ the directions across and along the layers and with 〈.) the volume average $\left[\langle f\rangle=\theta f^{-}+(1-\theta) f^{+}\right.$with $\theta$ the filling fraction of the layers, Fig. 1]. In this study, we show that the actual anisotropy due to a layered structuration of the bathymetry is much larger than the one predicted by (2). Indeed, even if the SWE (1) is valid for wavelengths much larger than the depth, it is unable to account for the near field effects in a subwavelength structure. In order to properly model these effects, the homogenization of the full 3D linear water wave problem needs to be considered. Following Ref. [14], we shall see that the effective water depths $\left(h w_{\mathrm{x}}, h_{\mathrm{y}}\right)$ are of the form

$$
\text { 3D problem } \quad h_{\mathrm{x}}=\ell \int_{\mathrm{Y}} \frac{\partial \Phi}{\partial x_{\mathrm{r}}}, \quad h_{\mathrm{y}}=\langle h\rangle,
$$

where $\ell$ is the periodicity of the bathymetry and $\Phi$ is the velocity potential associated to a simple potential flow problem, see (11). Comparing (2) to (3), it appears that only $h_{\mathrm{x}}$ is impacted by 3D effects and we shall see that $h_{\mathrm{x}}$ in (3) may be much smaller than $\left\langle h^{-1}\right\rangle^{-1}$. The important consequence is that the resulting anisotropy of the effective medium can be much larger than the classical homogenization of layered media, due to 3D near field effects.

The homogenized model of the linear 3D wave problem is presented in Sec. II; it is shown that the price to pay for the effective depth $h_{\mathrm{x}}$ in (3) is the resolution of a static problem on $\Phi$ which can be done once and for all (a simple script to do so is provided in the Appendix A). The validation of the model is presented in Sec. III. We built a metamaterial structure in laboratory experiments and inspected the anisotropic propagation of water waves over such a structure. First, a Floquet-Bloch analysis of the 3D linear problem is presented which allows us to validate the model in the very low frequency regime and to propose an

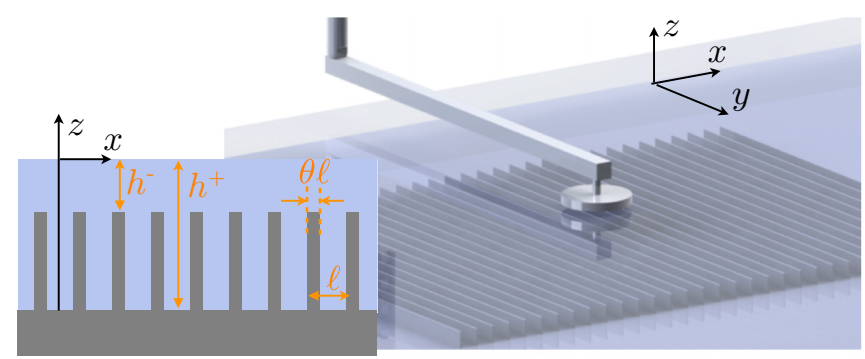

FIG. 1. Metamaterial for water waves, consisting of a subwavelength layered variation of the bathymetry. In the experiments, a thin tip excites water waves propagating over the metamaterial structure. 
anisotropic version of the dispersion relation to account for dispersive effects. The experimental measurements confirm the accuracy of the model and show that the linear theory is robust with respect to nonlinear and viscous effects. Finally, the anisotropy variations are inspected as a function of the geometrical parameters.

\section{HOMOGENIZATION OF THE 3D WATER WAVE PROBLEM}

The homogenization of the full 3D water wave problem has been first presented in Ref. [14]; in this paper, the authors conducted the homogenization to a high order so as to capture the first nonlinear and dispersive contributions. Later on the problem has been revisited using similar techniques in Refs. [15,16].

In this section, we shall derive a homogenized model similar to that proposed in Ref. [14] which allows us to identify the potential flow problem for $\Phi$ in (3). We consider the harmonic regime with time dependence $e^{-i \omega t}$. Using the assumptions of an inviscid, incompressible fluid and an irrotational flow, the velocity potential $\varphi(\mathbf{r}, z)$ satisfies

$$
\begin{aligned}
& \Delta \varphi=0, \\
& \frac{\partial \varphi}{\partial z}=\frac{\omega^{2}}{g} \varphi, \quad \text { at } z=0, \\
& \nabla \varphi \cdot \boldsymbol{n}=0, \quad \text { on } \Gamma,
\end{aligned}
$$

with $\mathbf{r}=(x, y)$ the coordinates in the horizontal plane, $z$ the vertical coordinate, and $t$ the time; $z=0$ refers to the unperturbed free surface position and $\Gamma$ the nonflat bottom (and $\boldsymbol{n}$ is the vector normal to $\Gamma$ ). Then, the displacement of the free surface $\eta(\mathbf{r})$ is linked to the velocity potential through the relation

$$
\eta(\mathbf{r})=\frac{i \omega}{g} \varphi(\mathbf{r}, 0) .
$$

The bottom $\Gamma$ is a patterned surface, resulting in sudden variations in the water depths $\left(h^{+}, h^{-}\right)$with spacing $\ell$ [Fig. 2(a)], and we consider $\ell$ of the same order of magnitude than $\left(h^{+}, h^{-}\right)$. In the low frequency regime the parameter

$$
\varepsilon=\omega \sqrt{\frac{\ell}{g}} \ll 1
$$

measures the small spacing of the microstructure compared to the wavelength, and it is also a shallowness parameter.

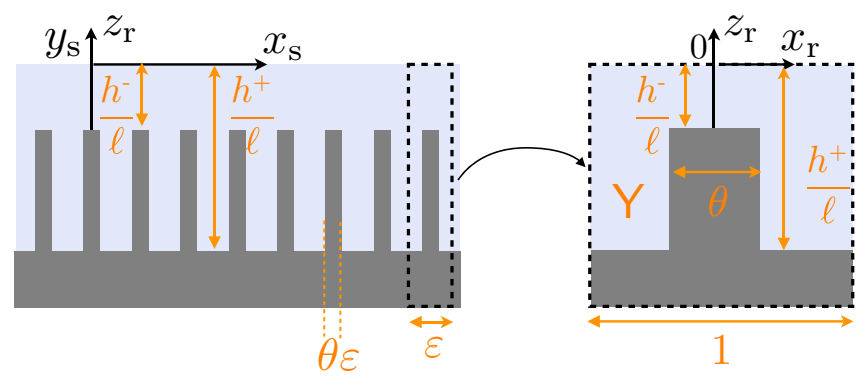

FIG. 2. Three-dimensional configuration of the layered sea bed in $\left(\mathbf{r}_{\mathrm{s}}, z_{\mathrm{r}}\right)$ coordinates and a zoom on the two-dimensional unit cell in $\left(x_{\mathrm{r}}, z_{\mathrm{r}}\right)$ coordinates.
The asymptotic analysis is performed owing to the two typical length scales separated by $\varepsilon$, the microscopic scale $\ell$ of the microstructure and the macroscopic scale $\sqrt{g \ell} / \omega$ of the wavelength. The separation of these scales is accounted for by defining $\varphi\left(\mathbf{r}_{\mathrm{s}}, x_{\mathrm{r}}, z_{\mathrm{r}}\right)$, with $\mathbf{r}_{\mathrm{s}}=\left(x_{\mathrm{s}}, y_{\mathrm{s}}\right)$ and

$$
\mathbf{r}_{\mathrm{s}}=\frac{\omega}{\sqrt{g \ell}} \mathbf{r},
$$

and with

$$
\left(x_{\mathrm{r}}, z_{\mathrm{r}}\right)=\left(\frac{x}{\ell}, \frac{z}{\ell}\right) .
$$

The macroscopic coordinate $\mathbf{r}_{\mathrm{s}}$ is adapted to describe the slow variations of the surface waves propagating at the free surface, and the microscopic coordinate $\left(x_{\mathrm{r}}, z_{\mathrm{r}}\right)$ is adapted to describe the rapid variations of the potential along $x_{\mathrm{r}}$ due to the microstructuration and in the vertical direction because of the shallowness regime (see the rescaled unit cell in Fig. 2).

Due to this separation of scale, (4) now reads as

$$
\begin{aligned}
& \Delta_{\mathrm{r}} \varphi+2 \varepsilon \frac{\partial^{2} \varphi}{\partial x_{\mathrm{s}} \partial x_{\mathrm{r}}}+\varepsilon^{2} \Delta_{\mathrm{s}} \varphi=0, \\
& \frac{\partial \varphi}{\partial z_{\mathrm{r}}}=\varepsilon^{2} \varphi, \quad \text { at } z_{\mathrm{r}}=0, \\
& \nabla_{\mathrm{r}} \varphi \cdot \boldsymbol{n}=-\varepsilon \frac{\partial \varphi}{\partial x_{\mathrm{s}}} n_{x}, \quad \text { on } \Gamma, \\
& \varphi \text { and } \frac{\partial \varphi}{\partial x_{\mathrm{r}}} \text { periodic } w . r . t . x_{\mathrm{r}},
\end{aligned}
$$

with $n_{x}=\boldsymbol{n} \cdot \mathbf{e}_{x}$. We shall establish the equation satisfied by $\varphi$ at the dominant order in the expansion

$$
\varphi=\varphi^{0}\left(\mathbf{r}_{\mathrm{s}}, x_{\mathrm{r}}, z_{\mathrm{r}}\right)+\varepsilon \varphi^{1}\left(\mathbf{r}_{\mathrm{s}}, x_{\mathrm{r}}, z_{\mathrm{r}}\right)+\varepsilon^{2} \varphi^{2}\left(\mathbf{r}_{\mathrm{s}}, x_{\mathrm{r}}, z_{\mathrm{r}}\right) \ldots
$$

At the order $\varepsilon^{0},(6)$ reduces to

$$
\begin{aligned}
& \Delta_{\mathrm{r}} \varphi^{0}=0, \\
& \frac{\partial \varphi^{0}}{\partial z_{\mathrm{r}}}=0, \quad \text { at } z_{\mathrm{r}}=0, \\
& \nabla_{\mathrm{r}} \varphi^{0} \cdot \boldsymbol{n}=0, \quad \text { on } \Gamma, \\
& \varphi^{0} \text { and } \frac{\partial \varphi^{0}}{\partial x_{\mathrm{r}}} \text { periodic w.r.t. } x_{\mathrm{r}} .
\end{aligned}
$$

It is easy to see that $\nabla_{\mathrm{r}} \varphi^{0}=0$, from which $\varphi^{0}\left(\mathbf{r}_{\mathrm{s}}\right)$ depends on the macroscopic coordinates only. Next, at the order $\varepsilon$, (6) yields

$$
\begin{aligned}
& \Delta_{\mathrm{r}} \varphi^{1}=0, \\
& \frac{\partial \varphi^{1}}{\partial z_{\mathrm{r}}}=0, \quad \text { at } z_{\mathrm{r}}=0, \\
& \nabla_{\mathrm{r}} \varphi^{1} \cdot \boldsymbol{n}=-\frac{\partial \varphi^{0}}{\partial x_{\mathrm{s}}}\left(\mathbf{r}_{\mathrm{s}}\right) n_{x}, \quad \text { on } \Gamma, \\
& \varphi^{1} \text { and } \frac{\partial \varphi^{1}}{\partial x_{\mathrm{r}}} \text { periodic w.r.t. } x_{\mathrm{r}} .
\end{aligned}
$$

The above problem on $\varphi^{1}$ is linear w.r.t., the source term $\partial_{x_{\mathrm{s}}} \varphi^{0}\left(\mathbf{r}_{\mathrm{s}}\right)$ being independent of $\left(x_{\mathrm{r}}, z_{\mathrm{r}}\right)$. Thus, we can set

$$
\varphi^{1}\left(\mathbf{r}_{\mathrm{s}}, x_{\mathrm{r}}, z_{\mathrm{r}}\right)=\frac{\partial \varphi^{0}}{\partial x_{\mathrm{s}}}\left(\mathbf{r}_{\mathrm{s}}\right)\left(\Phi\left(x_{\mathrm{r}}, z_{\mathrm{r}}\right)-x_{\mathrm{r}}\right)+\hat{\varphi}^{1}\left(\mathbf{r}_{\mathrm{s}}\right)
$$


with $\Phi$ satisfying the so-called "cell problem" defined at the microscopic scale

$$
\begin{aligned}
& \Delta \Phi=0, \\
& \frac{\partial \Phi}{\partial z_{\mathrm{r}}}=0, \quad \text { at } z_{\mathrm{r}}=0, \\
& \nabla \Phi \cdot \boldsymbol{n}=0, \quad \text { on } \Gamma, \\
& \Phi\left(\frac{1}{2}, z_{\mathrm{r}}\right)=\Phi\left(-\frac{1}{2}, z_{\mathrm{r}}\right)+1, \\
& \frac{\partial \Phi}{\partial x_{\mathrm{r}}}\left(\frac{1}{2}, z_{\mathrm{r}}\right)=\frac{\partial \Phi}{\partial x_{\mathrm{r}}}\left(-\frac{1}{2}, z_{\mathrm{r}}\right),
\end{aligned}
$$

and the last conditions are identical to the conditions $\left(\Phi-x_{\mathrm{r}}\right)$ and $\partial_{x_{\mathrm{r}}} \Phi$ periodic w.r.t. $x_{\mathrm{r}}$ as $\varphi^{1}$ and $\partial_{x_{\mathrm{r}}} \varphi^{1}$ do.

Equation (11) is the only cell problem that has to be solved in order to obtain the effective medium wave equation (3). It is worth noting that this problem is independent of the frequency and that it corresponds to the problem of a potential flow in a corrugated rigid duct. Note also that we defined $\hat{\varphi}^{1}\left(\mathbf{r}_{\mathrm{s}}\right)$ in (10) since the problem (9) on $\varphi^{1}$ is defined up to a function of $\mathbf{r}_{\mathrm{s}}$; this is incidental at the leading order but would be of importance for the higher order solution.

The last step consists of integrating over the unit cell $Y$ (Fig. 2) the first equation in (6) written at the order $\varepsilon^{2}$, specifically

$$
\int_{Y}\left(\Delta_{\mathrm{r}} \varphi^{2}+2 \frac{\partial^{2} \varphi^{1}}{\partial x_{\mathrm{r}} \partial x_{\mathrm{S}}}+\Delta_{\mathrm{S}} \varphi^{0}\right) \mathrm{d} x_{\mathrm{r}} \mathrm{d} z_{\mathrm{r}}=0 .
$$

Using the boundary conditions (6) satisfied by $\varphi^{2}$ at $z_{\mathrm{r}}=0$ and on $\Gamma$ along with (10), we get

$$
\begin{array}{r}
\int_{\mathrm{Y}} \Delta_{\mathrm{r}} \varphi^{2} \mathrm{~d} x_{\mathrm{r}} \mathrm{d} z_{\mathrm{r}}=\varphi^{0}\left(\mathbf{r}_{\mathrm{s}}\right)+\frac{\partial^{2} \varphi^{0}}{\partial x_{\mathrm{s}}^{2}}\left(\mathbf{r}_{\mathrm{s}}\right) \int_{\Gamma}\left(\Phi-x_{\mathrm{r}}\right) n_{x} \mathrm{~d} s, \\
\int_{\mathrm{Y}} \frac{\partial^{2} \varphi^{1}}{\partial x_{\mathrm{r}} \partial x_{\mathrm{s}}} \mathrm{d} x_{\mathrm{r}} \mathrm{d} z_{\mathrm{r}}=\frac{\partial^{2} \varphi^{0}}{\partial x_{\mathrm{s}}^{2}}\left(\mathbf{r}_{\mathrm{s}}\right) \int_{\mathrm{Y}}\left(\frac{\partial \Phi}{\partial x_{\mathrm{r}}}-1\right) \mathrm{d} x_{\mathrm{r}} \mathrm{d} z_{\mathrm{r}} .
\end{array}
$$

It is now sufficient to use that $\int_{Y}\left(\partial_{x_{\mathrm{r}}} \Phi-1\right) \mathrm{d} x_{\mathrm{r}} \mathrm{d} z_{\mathrm{r}}+\int_{\Gamma}(\Phi-$ $\left.x_{\mathrm{r}}\right) n_{x} \mathrm{~d} s=0$ from (11), and that the surface of the unit cell is $\int_{\mathrm{Y}} \mathrm{d} x_{\mathrm{r}} \mathrm{d} z_{\mathrm{r}}=\langle h\rangle / \ell$ [with $\left.\langle h\rangle=\theta h^{-}+(1-\theta) h^{+}\right]$. Doing so, we get that

$$
\frac{h_{\mathrm{x}}}{\ell} \frac{\partial^{2} \varphi^{0}}{\partial x_{\mathrm{s}}^{2}}+\frac{h_{\mathrm{y}}}{\ell} \frac{\partial^{2} \varphi^{0}}{\partial y_{\mathrm{s}}^{2}}+\varphi^{0}=0 .
$$

Once coming back to the real space, we find the equation satisfied by $\varphi \sim \varphi^{0}$ in $(x, y)$ coordinate, thus satisfied by $\eta$, of the form

$$
h_{\mathrm{x}} \frac{\partial^{2} \eta}{\partial x^{2}}+h_{\mathrm{y}} \frac{\partial^{2} \eta}{\partial y^{2}}+\frac{\omega^{2}}{g} \eta=0
$$

with $\left(h_{\mathrm{x}}, h_{\mathrm{y}}\right)$ given by (3). The determination of $h_{\mathrm{x}}$ has to be done by solving (11); this can be done numerically easily with any solver and we provide in Appendix A a way to do so, by means of a multimodal method.

\section{VALIDATION OF THE METAMATERIAL ANISOTROPY}

We built a metamaterial structure as shown in Fig. 1 using plates of glass of $0.1 \mathrm{~cm}$ large, $7.5 \mathrm{~cm}$ long, and $1 \mathrm{~cm}$ height.
The plates were arranged on the bottom of a laboratory tank along the $x$ and $y$ directions in order to form a metamaterial structure with the following characteristics: layer spacing $\ell=$ $0.6 \mathrm{~cm}$, filling fraction $\theta=1 / 6$, and water depths $h^{-}=0.4 \mathrm{~cm}$, $h^{+}=1.4 \mathrm{~cm}$. The whole structure comprised of 60 layers covers a region of $24 \mathrm{~cm} \times 36 \mathrm{~cm}$.

In this configuration, from (3), and the numerical resolution of (11), we get

$$
h_{\mathrm{x}}=0.48 \mathrm{~cm}, \quad h_{\mathrm{y}}=1.23 \mathrm{~cm} .
$$

The effective depth $h_{\mathrm{y}}=\langle h\rangle$ is close to $h^{+}$which is expected for our thin layers $(\theta=1 / 6)$. Also, $h_{\mathrm{x}}$ is close to $h^{-}$, and this is intuitive as well since the layers are relatively close to each other. Thus, the flow associated to waves propagating across the layers cannot enter deeply between two layers, and it remains confined at depths of the order of $h^{-}$, i.e., mainly sensitive to the smaller depth. In comparison, the homogenized version of the approximate shallow water equation, unable to account for the near field between two layers, yields a less intuitive value of $h_{\mathrm{x}}=\left\langle h^{-1}\right\rangle^{-1}=1 \mathrm{~cm}$, from (2).

\section{A. Floquet analysis}

To compute the Floquet-Bloch spectrum, we solve the full 3D problem (4) with Bloch condition in the unit cell Y. Owing to the invariance of the geometry along $y$, this reduces in practice to a $2 \mathrm{D}$ problem with

$$
\begin{aligned}
\varphi(x+\ell, y, z) & =e^{i k_{x} \ell} \varphi(x, y, z), \\
\frac{\partial \varphi}{\partial x}(x+\ell, y, z) & =e^{i k_{x} \ell} \frac{\partial \varphi}{\partial x}(x, y, z),
\end{aligned}
$$

with a dependence on $y$ being simply of the form $e^{i k_{y} y}$. The problem is solved for each frequency $\omega$ and each wave number $k_{y}$ to get $k_{x}$, and we report in Appendix B a numerical

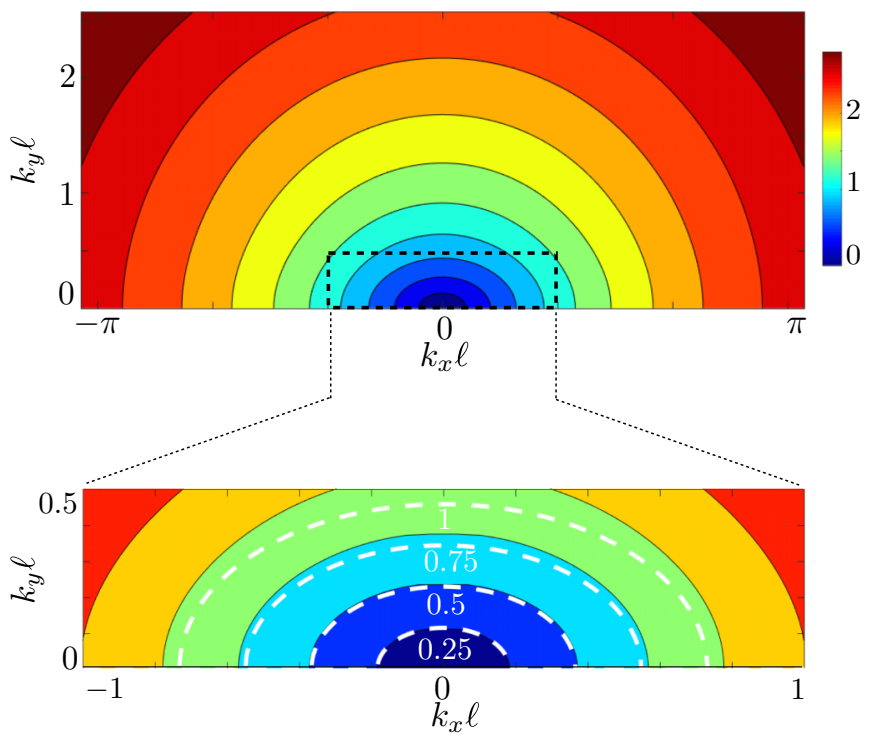

FIG. 3. Band structure $\omega \sqrt{g / h^{+}}$of the layered metamaterial against $\left(k_{\mathrm{x}} \ell, k_{\mathrm{y}} \ell\right)$ given by the Floquet-Bloch analysis of the $3 \mathrm{D}$ problem. The inset at bottom shows a zoom for $\omega \sqrt{g / h^{+}}<1$ and in dashed white lines, the 3D homogenized prediction, (18) with (16) for $\omega \sqrt{g / h^{+}}=0.25,0.5,0.75$, and 1 . 


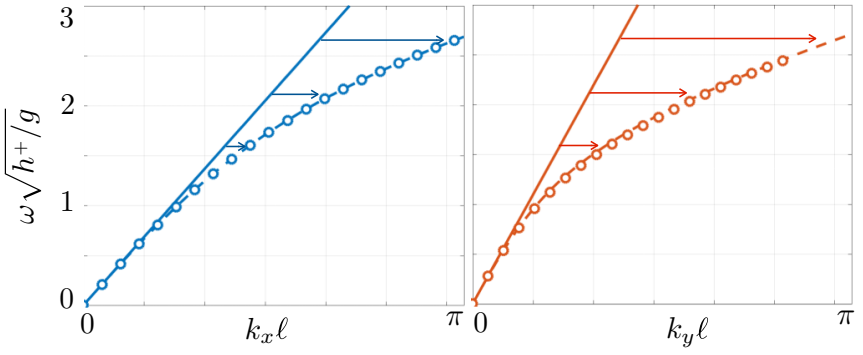

FIG. 4. Nondimensional wave numbers $k_{\mathrm{x}} \ell$ and $k_{\mathrm{y}} \ell$ as a function of $\omega \sqrt{g / h^{+}}$; open symbols: obtained from the Floquet-Bloch analysis, plain lines: from the $3 \mathrm{D}$ homogenized prediction in the shallow water regime, Eq. (18), and dashed lines: including the dispersive correction (19) [in both cases, with $\left(h_{\mathrm{x}}, h_{\mathrm{y}}\right)$ in (16)]. The arrows show the dispersive correction.

procedure to do it using a multimodal method. We computed the band structure of our metamaterial and the result is shown in Fig. 3. From the zoom at low frequency $\omega \sqrt{g / h^{+}}<1$ in Fig. 3, the dispersion relation

$$
\frac{\omega^{2}}{g}=h_{\mathrm{x}} k_{\mathrm{x}}^{2}+h_{\mathrm{y}} k_{\mathrm{y}}^{2}
$$

predicted by the homogenized model (15) [with $h_{\mathrm{x}}$ and $h_{\mathrm{y}}$ in (16)] is found to reproduce with good accuracy the actual band structure.

However, under scrutiny of Fig. 3(a), it is visible that the anisotropy decreases slightly when increasing the frequency, which is attributable to dispersive effects. Interestingly, for still unknown reasons, it turns out that these dispersive effects can be described by extending heuristically the dispersion relation for constant water depth to its anisotropic version

$$
\omega^{2}=g\left(k_{\mathrm{x}}^{2} h_{\mathrm{x}}+k_{\mathrm{y}}^{2} h_{\mathrm{y}}\right) \tanh (k h) / k h, \quad k h=\sqrt{k_{\mathrm{x}}^{2} h_{\mathrm{x}}^{2}+k_{\mathrm{y}}^{2} h_{\mathrm{y}}^{2}} .
$$

This is illustrated in Fig. 4 where we reported $\left(k_{\mathrm{x}}, k_{\mathrm{y}}\right)$ calculated using (18) (plain lines) and using (19) (dashed lines), compared to the $\left(k_{\mathrm{x}}, k_{\mathrm{y}}\right)$ given by the Floquet-Bloch analysis (symbols). The dispersive correction given by (19) appears to be efficient in the whole Brillouin region. This surprising result allows us to use $\left(h_{\mathrm{x}}, h_{\mathrm{y}}\right)$, obtained from low frequency homogenization, to characterize the response of the metamaterial both in the nondispersive and dispersive regimes. More specifically, we found that the nondispersive dispersion relation (18) has an error, when compared to the Floquet-Bloch solution, of $20 \%$ for $\omega \sqrt{g / h^{+}}<1$ and $90 \%$ for $\omega \sqrt{g / h^{+}}<2$; accounting for (19) reduces the error to $1 \%$ for $\omega \sqrt{g / h^{+}}<1$ and $4 \%$ for $\omega \sqrt{g / h^{+}}<2$. Higher order homogenization as done in Ref. [14] might help to understand this dispersive behavior.

\section{B. Experimental results}

To inspect the anisotropy of our structure, a point source is realized using a linear motor equipped with a thin tip moving vertically in a sinusoidal motion; the source emits from the center of the structure. Our working frequency range is $f \in[2,6] \mathrm{Hz}$, whence $\omega \sqrt{h^{+} / g}$ ranges in $[0.5,1.4]$. The wave fields are measured using an optical method (Fourier
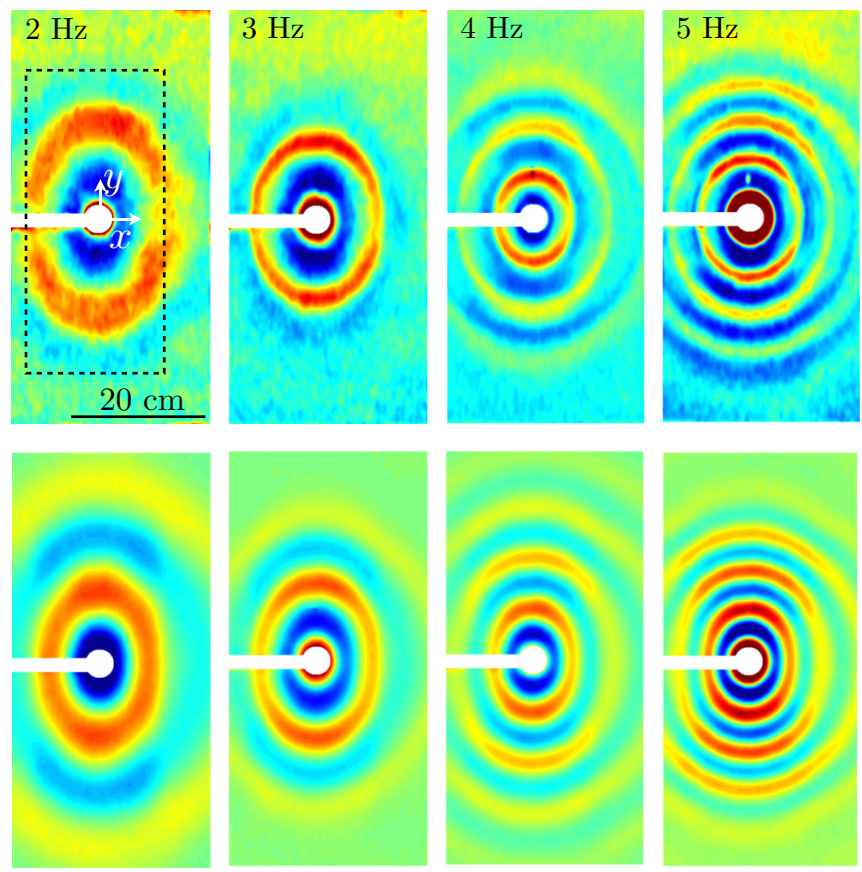

FIG. 5. Experimental measurements of the surface wave elevation due to a point source at the center of the metamaterial structure. Top panels: instantaneous snapshots $\eta(\mathbf{r}, t)$ measured by FTP (the dotted lines at $2 \mathrm{~Hz}$ show the boundaries of the metamaterial). Bottom panels: corresponding real parts of the Fourier component at $f$ of the wave field $\hat{\eta}(\mathbf{r}, f),(20)$.

transform profilometry, termed FTP below) able to achieve space-time resolved measurement of the free surface elevation $[17,18]$. The measurement domain is $34 \times 60 \mathrm{~cm}^{2}$ with a pixel size of $0.68 \mathrm{~mm}$. The temporal resolution is given by the frame rate of the camera, which is fixed at $125 \mathrm{~Hz}$ in our experiments. Typical instantaneous fields of the free surface elevation $\eta(\mathbf{r}, t)$ measured by FTP are reported in Fig. 5 (top panels). The anisotropy of the structure is visible to the necked eye, particularly at the lowest frequencies.

To be more quantitative, the wavelengths $\lambda_{x}$ and $\lambda_{y}$ have been measured. We benefit from the space-time resolution in

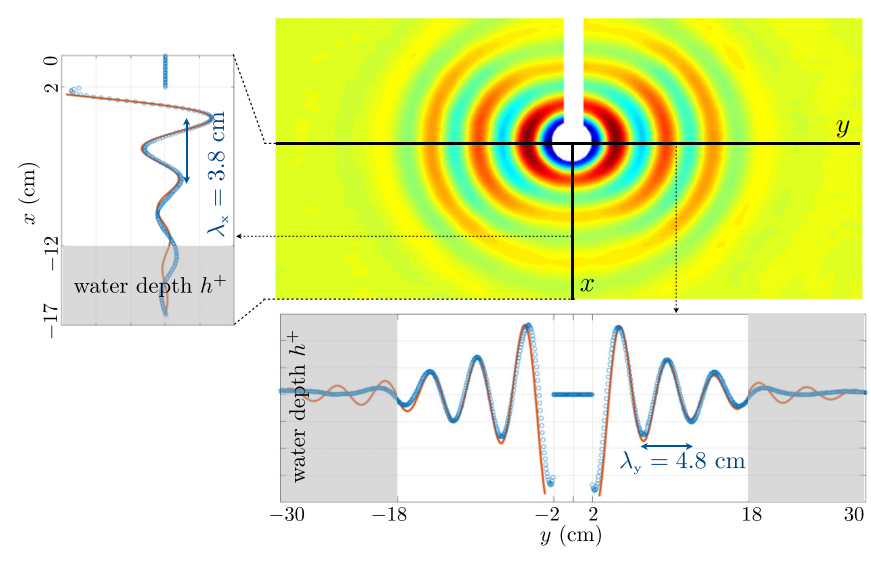

FIG. 6. The main plot shows the real part of $\hat{\eta}(\mathbf{r}, f)$ computed with (20) from the experimental measurements at $f=5.5 \mathrm{~Hz}$; the insets show the profiles at $x=0$ and $y=0$ (blue symbols) along with the best fits with Hankel function $H^{0}\left(k_{\mathrm{x}} x\right)$ and $H^{0}\left(k_{\mathrm{y}} y\right)$ (red curves). 


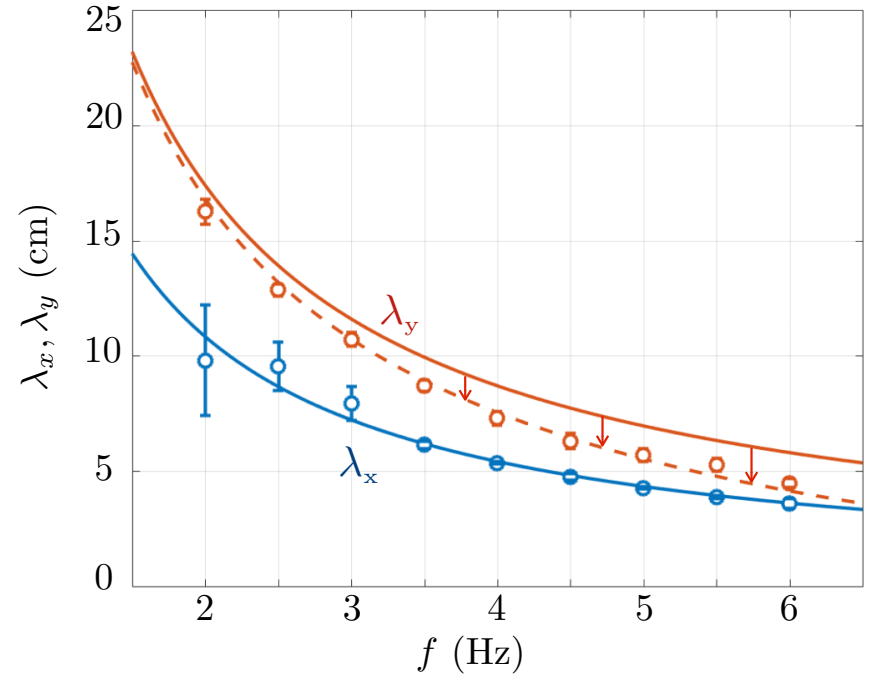

FIG. 7. Wave numbers $\left(\lambda_{x}, \lambda_{y}\right)$ against the frequency, determined from the experimental measurements (symbols) and given by the $3 \mathrm{D}$ homogenization prediction: in the shallow water regime (18) with (16) (plain lines) and including the dispersive correction (19) (dashed lines).

our measurements to compute the fields of the complex Fourier components $\hat{\eta}(\mathbf{r}, n f), n=0,1, \ldots$, defined as

$$
\hat{\eta}(\mathbf{r}, n f)=\frac{1}{T} \int_{0}^{T} \eta(\mathbf{r}, t) e^{-2 i \pi n f t} \mathrm{~d} t,
$$

with $T=1 / f$. In practice, a temporal signal $t \in\left(0, t_{f}\right)$ is used, with $t=0$ chosen after the signal emitted by the source has reached the boundaries of our metamaterial structure and with $t_{f}$ chosen in order to avoid the spurious wave reflections at the walls of the tank. Next, the interval $\left(0, t_{f}\right)$ is taken back in $(0, T)$ using $t \rightarrow t-n T$, with $n$ the floor part of $(t / T)$. The complex fields $\hat{\eta}(\mathbf{r}, f)$ at the fundamental frequency $f$ have been computed, and we report their real parts in Fig. 5 (bottom panels). With maximum amplitudes of the waves about 1 $\mathrm{cm}$ near the source, the contribution of the higher harmonics (essentially at $2 f$ ) were found to be of the order of $15 \%$ on average.

The determination of the wavelengths $\left(\lambda_{\mathrm{x}}, \lambda_{\mathrm{y}}\right)$ has been done on $\hat{\eta}(\mathbf{r}, f)$ along the lines $y=0$ and $x=0$, respectively, by means of a measure of peak-to-peak distances and by means of a fit by a Hankel function. A typical example is shown in Fig. 6 at $f=5.5 \mathrm{~Hz}$, where we reported the experimental profiles on the centerlines $x=0$ and $y=0$ and the corresponding best fits with a Hankel function $H^{0}\left(k_{\mathrm{x}} x\right)$ and $H^{0}\left(k_{\mathrm{y}} y\right)$, respectively.

The wavelengths $\left(\lambda_{\mathrm{x}}, \lambda_{\mathrm{y}}\right)$ have been determined at each frequency $f \in(2,6) \mathrm{Hz}$ with a spacing of $0.5 \mathrm{~Hz}$. Figure 7 reports the main result of our study. Together with the experimental measurements (symbols), we show the effective wavelengths given by the $3 \mathrm{D}$ homogenization prediction in the shallow water regime (18) and including the dispersive correction (19), in both cases using the homogenized prediction (16).

The homogenized model in the shallow water is already accurate for the smallest wavelength $\lambda_{\mathrm{x}}$ which confirms that the effective depth has to be calculated considering 3D effects. Next $\lambda_{y}$ departs slightly from the average predicted in the shallow water regime, and the shift is corrected efficiently considering the dispersive correction proposed in (19).

\section{Maximum anisotropy in metamaterial structure}

We have seen that the anisotropy of a patterned bottom is higher than that expected by simple inspection of the shallow water equation, and reasonable questions are: How high can be the actual anisotropy? On which parameters does it depend? To answer these questions, we define a measure of the anisotropy by the parameter $\mathcal{A}$

$$
\mathcal{A} \equiv\left(\frac{\lambda_{\mathrm{y}}}{\lambda_{\mathrm{x}}}\right)^{2} \simeq \frac{h_{\mathrm{y}}}{h_{\mathrm{x}}},
$$

where the approximation $\mathcal{A} \simeq h_{\mathrm{y}} / h_{\mathrm{x}}$, valid at low frequencies, is used to avoid an additional discussion on the frequency dependence.

We kept the same water depths as in our experiments $\left(h^{+}=1.4 \mathrm{~cm}, h^{-}=0.4 \mathrm{~cm}\right)$ and we considered a large spacing
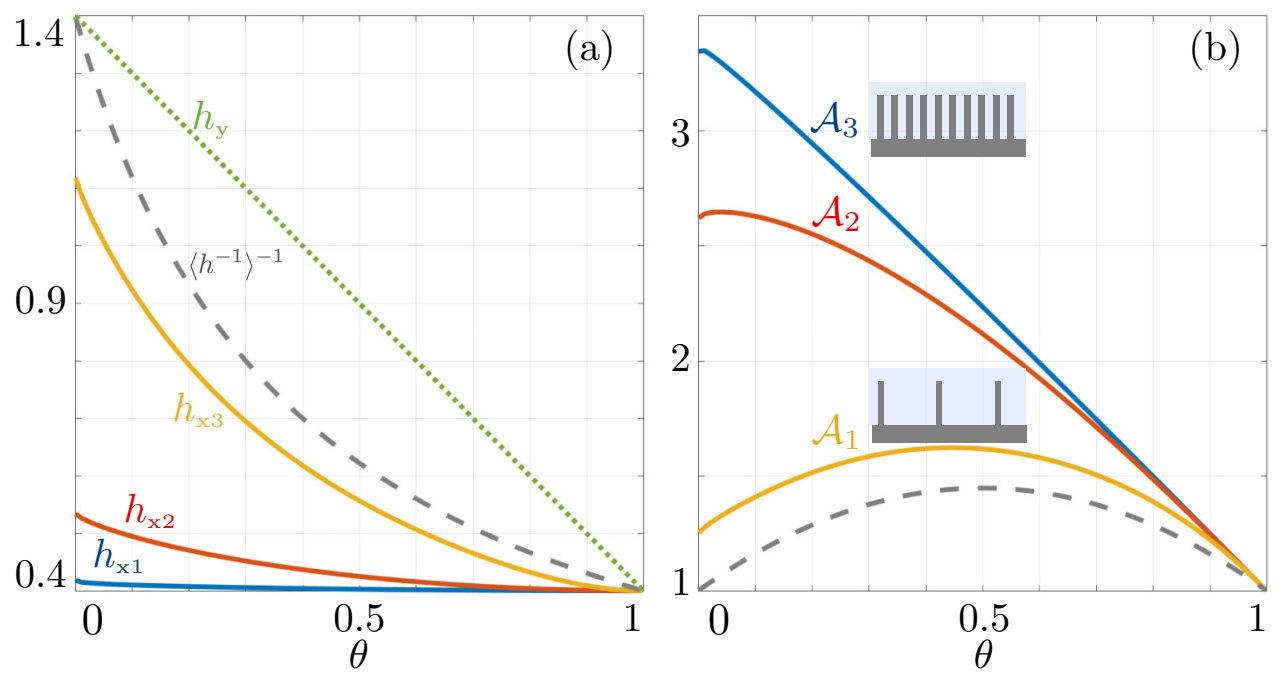

FIG. 8. (a) Variation of $h_{\mathrm{x}}$ (in cm) as a function of $\theta$ for $\ell / h^{+}=0.04\left(h_{\mathrm{x} 1}\right), \ell / h^{+}=0.4\left(h_{\mathrm{x} 2}\right)$, and $\ell / h^{+}=4\left(h_{\mathrm{x} 3}\right) ; h_{\mathrm{y}}$ is independent on $\ell / h^{+}$. (b) Resulting anisotropy $\mathcal{A}$. In (a) and (b) the dashed gray lines show the 2D SWE predictions, using $h_{\mathrm{x}}=\left\langle h^{-1}\right\rangle^{-1}$, for comparison. 
$\ell / h^{+}=4$, a small spacing $\ell / h^{+}=0.04$, and the intermediate one $\ell / h^{+}=0.4$ being that or our experiments. Figure 8 shows the graphs of $\left(h_{\mathrm{x}}, h_{\mathrm{y}}\right)$, from (3), against $\theta$ in these three cases and the resulting anisotropy $\mathcal{A}\left(h_{\mathrm{x}}\right.$ has been calculated using the script given in the Appendix A). For comparison, the 2D SWE predictions are reported, with $h_{\mathrm{x}}=\left\langle h^{-1}\right\rangle^{-1}$, and it is worth noting that $h_{\mathrm{x}}$ and $\mathcal{A}$ are independent of $\ell$ in this case. It appears the maximum allowed value $\mathcal{A}=h^{+} / h^{-}$is obtained decreasing the layer spacing $\ell$ and the layer thickness $(\theta=0)$, i.e., the maximum anisotropy is reached for very tight thin plates. For such compact array, $h_{\mathrm{x}}$ remains close to $h^{-}$for any $\theta$ [see $h_{\mathrm{x} 1}$ in Fig. 8(a)]. This is quite intuitive since the flow cannot enter in the dense region of vertical layers which thus becomes similar to a flat bottom at depth $h^{-}$. Meanwhile, decreasing $\theta$ produces a linear increase in $h_{\mathrm{y}}$, with $h_{\mathrm{y}}=h^{-}$ (whence $\mathcal{A}=1$ ) at $\theta=1$ to $h_{\mathrm{y}}=h^{+}$at $\theta=0$. This is the linear behavior that is observed for $\mathcal{A}_{1}\left(\ell / h^{+}=0.04\right)$, which simply reflects the linear decrease of $h_{\mathrm{y}}$ for an almost constant $h_{\mathrm{x}}$. The other limit for large $\ell / h^{+}$is instructive as well; in this case, the near field effects diminish, being confined in the vicinity of each isolated vertical layer. This is why the anisotropy approaches the approximate $2 \mathrm{D}$ shallow water result.

\section{CONCLUDING REMARKS}

In conclusion, we report good news from full 3D homogenization theory. When designing an anisotropic metamaterial for water waves, it might be tempting to evaluate the needed microstructure by solving the approximate $2 \mathrm{D}$ shallow water equation. Two drawbacks on this approach can be highlighted: (i) even for moderate anisotropy, the predicted microstructure requires bathymetry with high contrasts that are in general challenging to realize and (ii) the approximation of the model is questionable, all the more since we have very steep bathymetry. By applying the homogenization method to the full 3D linear water wave equation, it appears that these two drawbacks fall simultaneously. Besides, experimental results have shown that this linear theory is robust with respect to nonlinear and viscous effects. We believe that our findings will facilitate the design of metamaterials to control energy flow of water waves.

\section{ACKNOWLEDGMENTS}

A.M, P.P., and V.P. acknowledge the financial support of the Agence Nationale de la Recherche through the Grant No. DYNAMONDE ANR-12-BS09-0027-01. A.M. and J.-J.M. acknowledge the financial support of the CNRS-MI through the Grant No. PomS/Defi Infinity.

\section{APPENDIX A: MATLAB SCRIPT TO COMPUTE THE EFFECTIVE WATER DEPTH $\boldsymbol{h}_{\mathrm{x}}$}

We provide below a simple script to compute $h_{\mathrm{x}}$, defined in (3) with $\Phi$ satisfying (11). This can be done easily using a multimodal method based on matched eigenfunction expansion that we describe below.

First, we use that (i) $(\Phi-x)$ and $\partial_{x} \Phi$ are periodic and (ii) $(\Phi-x)$ is odd up to a constant that can be chosen equal to zero [in this section, we use the notation $(x, z)$ instead of $\left(x_{\mathrm{r}}, z_{\mathrm{r}}\right)$ for simplicity]. This is because the boundary conditions

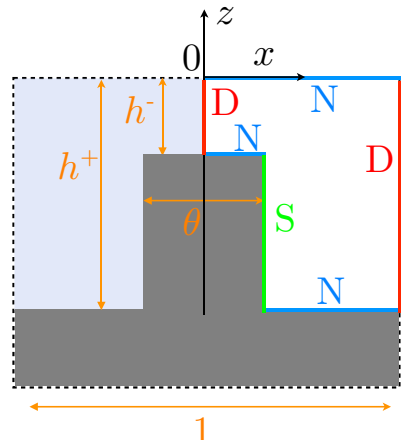

(a)

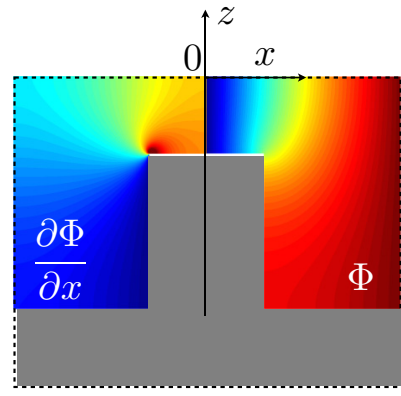

(b)
FIG. 9. (a) Unit cell in which the cell problem for $(\Phi-x)$ is defined. (b) Solution for the velocity potential $\Phi$ [shown for $x \in$ $(0,1 / 2)]$ and corresponding horizontal velocity $\partial_{x} \Phi$ [shown for $x \in$ $(-1 / 2,0)]$ whose integral provides $h_{\mathrm{x}}$.

on the vertical parts of $\Gamma$ at $x= \pm \theta / 2 \operatorname{read} \partial_{x}(\Phi-x)=-1$ (the boundary conditions on the horizontal parts of $\Gamma$ are of the Neumann type). Thus, the domain can be reduced to $x \in$ $(0,1 / 2)$ applying the boundary conditions for $(\Phi-x)$ (i) the free surface and the horizontal parts of the layers are associated to Neumann boundary condition ( $\mathrm{N}$ in Fig. 9), (ii) the parts of the domain at $x=0$ and $x=1 / 2$ are associated to Dirichlet boundary condition (D in Fig. 9), and only the vertical part of the layer at $x=\theta / 2$ is associated to a source term through $\partial_{x}(\Phi-x)=-1$ (condition S in Fig. 9).

The eigenfunction expansions are written in two the regions of constant depth, where two expansions of $\Phi$ are used; afterwards the continuity of $\Phi$ and of $\partial_{x} \Phi$ at $x=\theta / 2$ will be used. Specifically, we use the expansions

$$
\begin{aligned}
x \in & (0, \theta / 2): \\
\Phi(x, z)= & \sum_{n=1}^{N^{-}} B_{n} \frac{\sinh \left(a_{n}^{-} x\right)}{\sinh \left(a_{n}^{-} \theta / 2\right)} \Phi_{n}^{-}(z)+B_{0} x, \\
x \in & (\theta / 2,1 / 2): \\
\Phi(x, z)= & \sum_{n=1}^{N^{+}} A_{n} \frac{\sinh \left(a_{n}^{+}(x-1 / 2)\right)}{\sinh \left(a_{n}^{+}(\theta-1) / 2\right)} \Phi_{n}^{+}(z) \\
& +A_{0} x+\frac{1-A_{0}}{2},
\end{aligned}
$$

which already satisfy the Dirichlet boundary conditions for $(\Phi-x)$ at $x=0$ and $x=1 / 2$. With $a_{n}^{ \pm}=n \pi \ell / h^{ \pm}, n>0$, we define the normalized transverse functions

$$
\Phi_{n}^{ \pm}(z)=\sqrt{\frac{2}{h^{ \pm}}} \cos \left(a_{n}^{ \pm} z\right),
$$

which are adapted to the Neumann boundary conditions at $z=0$ and $z=-h^{ \pm} / \ell$. We have $\left(N^{+}+N^{-}+2\right)$ unknowns $\left(A_{n}, B_{n}\right)$ and we shall write $\left(N^{+}+N^{-}+2\right)$ equations to determine them. At $x=\theta / 2$, the continuity of $\Phi$ is accounted for by imposing

$$
\begin{aligned}
\int_{Y^{-}} \Phi^{-} \mathrm{d} z & =\int_{Y^{-}} \Phi^{+} \mathrm{d} z \\
\int_{Y^{-}} \Phi^{-} \Phi_{n}^{-}(z) \mathrm{d} z & =\int_{Y^{-}} \Phi^{+} \Phi_{n}^{-}(z) \mathrm{d} z
\end{aligned}
$$


where $Y^{ \pm}=\left(-h^{ \pm} / \ell, 0\right)$ and with the notations $f^{ \pm}=$ $f\left(\theta^{ \pm} / 2, z\right)$; this provides $\left(N^{-}+1\right)$ relations. Next, the continuity of $\partial_{x} \Phi$ is written as

$$
\begin{aligned}
\int_{Y^{-}} \frac{\partial \Phi^{-}}{\partial x} \mathrm{~d} z & =\int_{Y^{+}} \frac{\partial \Phi^{+}}{\partial x} \mathrm{~d} z \\
\int_{Y^{-}} \frac{\partial \Phi^{-}}{\partial x} \Phi_{n}^{+}(z) \mathrm{d} z & =\int_{Y^{+}} \frac{\partial \Phi^{+}}{\partial x} \Phi_{n}^{+}(z) \mathrm{d} z
\end{aligned}
$$

which provide $\left(N^{+}+1\right)$ relations. Note that in (A4) the Neumann boundary conditions $\partial_{x} \Phi=0$ for $z \in\left(-h^{+},-h^{-}\right)$ are accounted for implicitly. Once $\left(A_{0}, B_{0}\right)$ are known, it is straightforward to get that $h_{\mathrm{x}}$ in (3) is given by

$$
h_{\mathrm{x}}=h^{+}(1-\theta) A_{0}+h^{-} \theta B_{0},
$$

where $A_{0}$ and $B_{0}$ depend on $h^{+} / \ell, h^{-} / \ell$ and $\theta$. Below we provide a Matlab script with $\left(\ell, \theta, h^{+}, h^{-}, N^{+}, N^{-}\right)$inputs to compute $\left(h_{\mathrm{x}}, h_{\mathrm{y}}\right)$.

function $[\mathrm{hx}, \mathrm{hy}]=\mathrm{PbCell}(\mathrm{L}$, theta, hp, hm, Np, Nm)

$h p=h p / L ; h m=h m / L ; \% n o n$ dimensional form

No1 $=\operatorname{sqrt}(2 / \mathrm{hp}) ; \mathrm{No} 2=\operatorname{sqrt}(2 / \mathrm{hm})$

$a \mathrm{pp}=(1: \mathrm{Np}) * \mathrm{pi} / \mathrm{hp} ; \mathrm{aNm}=(1: \mathrm{Nm}) * \mathrm{pi} / \mathrm{hm}$;

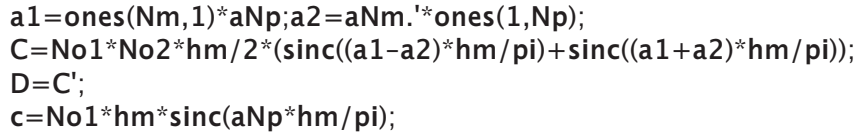

Source $=[\operatorname{zeros}(\mathrm{Nm}, 1) ; \mathrm{hm} / 2 ; \operatorname{zeros}(\mathrm{Np}+1,1)]$; Sol=Mat $\backslash$ Source;

$\mathrm{A}=\operatorname{Sol}(1: \mathrm{Np}) ; \mathrm{nn}=$ length $([\mathrm{A}])$;

$A 0=\operatorname{Sol}(n n+1: n n+1) ; n n=$ length $([A ; A 0])$;

$B=\operatorname{Sol}(n n+1: n n+N m) ; n n=$ length $([A ; A 0 ; B])$;

$\mathrm{BO}=\mathrm{Sol}(\mathrm{nn}+1: \mathrm{end})$

$h y=L^{*}\left(h m^{*}\right.$ theta $+h p^{*}(1-$ theta $\left.)\right) ; \%$ dimensional form $h x=L *(A 0 * h p *(1-$ theta $)+B 0 * h m *$ theta $)$;

\section{APPENDIX B: FLOQUET-BLOCH ANALYSIS OF THE FULL 3D PROBLEM}

The Floquet-Bloch analysis is performed in a cell of dimension $(-\ell / 2, \ell / 2) \times\left(-h^{+}, 0\right)$ (in the real space, see Fig. 10). To begin with, we compute the scattering matrix $S$ using a mode matching technique similar to the one presented in the previous section, see Ref. [19]. We use that

$$
\varphi(x, y, z)=e^{i k_{y} y} \tilde{\varphi}(x, z),
$$

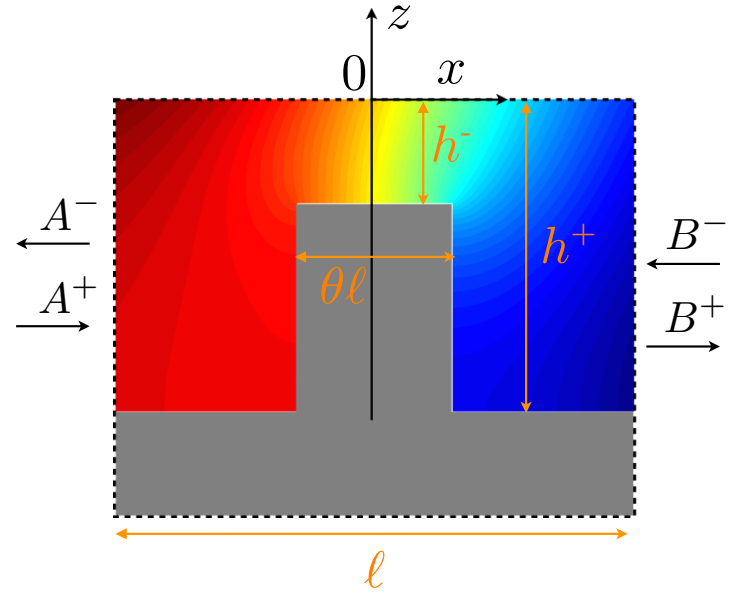

FIG. 10. Solution $\varphi(x, y=0, z)$ satisfying (4) with (17). $A^{ \pm}$ denote the vectors of the right/left going waves at $x=-\ell / 2$ and $B^{ \pm}$denote the vectors of the right/left going waves at $x=+\ell / 2$.

with

$$
\begin{aligned}
x & \leqslant-\ell / 2: \\
\tilde{\varphi}(x, z) & =\sum_{n=0}^{N}\left(A_{n}^{+} e^{i k_{n}(x+\ell / 2)}+A_{n}^{-} e^{-i k_{n}(x+\ell / 2)}\right) \varphi_{n}^{+}(z), \\
x & \geqslant \ell / 2: \\
\tilde{\varphi}(x, z) & =\sum_{n=0}^{N}\left(B_{n}^{+} e^{i k_{n}(x-\ell / 2)}+B_{n}^{-} e^{-i k_{n}(x-\ell / 2)}\right) \varphi_{n}^{+}(z),
\end{aligned}
$$

and

$$
\varphi_{n}^{+}(z)=\frac{\cosh \left(K_{n}\left(z+h^{+}\right)\right)}{\left[\frac{h}{2}\left(\sinh c\left(2 K_{n} h^{+}\right)+1\right)\right]^{1 / 2}} .
$$

The normalized transverse functions adapted to the boundary conditions of the Neumann type at $z=-h^{+}$and of the Robin type at $z=0$ if $K_{n}$ satisfies the dispersion relation of the water waves

$$
\omega^{2} / g=K_{n} \tanh \left(K_{n} h^{+}\right),
$$

and $\varphi$ satisfying the Laplace equation, we have

$$
k_{n}=\sqrt{K_{n}^{2}-k_{y}^{2}} \text {. }
$$

Then, similar expansions of $\varphi$ are written in $x \in(-\ell / 2,-$ $\theta \ell / 2),(-\theta \ell / 2, \theta \ell / 2)$ and, in $(\theta \ell / 2, \ell / 2)$ the expansions are written using the basis $\varphi_{n}^{-}(z)$ similar to (B3) with $h^{+} \rightarrow h^{-}$. Next, the matching of $\varphi$ and $\partial_{x} \varphi$ are applied at the junctions $x= \pm \ell / 2$ and $x= \pm \theta \ell / 2$, as in (A3)-(A4). This leaves us with with relations between the components $\left(A_{n}^{-}, B_{n}^{+}\right)$of the outgoing waves to the components $\left(A_{n}^{+}, B_{n}^{-}\right)$of the ingoing waves. Denoting $A^{+}=\left(A_{n}^{+}\right)$the vector of the components (the same for $A^{-}$and $B^{ \pm}$), these relations can be written

$$
\left(\begin{array}{l}
A^{-} \\
B^{+}
\end{array}\right)=S\left(\begin{array}{l}
A^{+} \\
B^{-}
\end{array}\right) \quad S=\left(\begin{array}{ll}
r & t \\
t & r
\end{array}\right),
$$

where we used that the cell is symmetric and centered at $x=0$, which simplifies the expression of $S$. The $S$ matrix involves the reflection $r$ and the transmission $t$ matrices which 
can be calculated in practice for $A^{+}=0$ (incident left going waves at $x=\ell / 2$ ) or for $B^{-}=0$ (incident right going wave at $x=-\ell / 2$ ). The system (B5) can be written as

$$
M_{1}\left(\begin{array}{l}
A^{+} \\
A^{-}
\end{array}\right)=M_{2}\left(\begin{array}{l}
B^{+} \\
B^{-}
\end{array}\right)
$$

where

$$
M_{1}=\left(\begin{array}{cc}
t & 0 \\
-r & I
\end{array}\right), \quad M_{2}=\left(\begin{array}{cc}
I & -r \\
0 & t
\end{array}\right)
$$

Projecting the expansions of $\varphi$ onto the $\varphi_{n}^{ \pm}$, it is easy to see that the Floquet-Bloch condition (17) reduces to

$$
\left(\begin{array}{l}
B^{+} \\
B^{-}
\end{array}\right)=\mu\left(\begin{array}{l}
A^{+} \\
A^{-}
\end{array}\right)
$$

with $\mu\left(\omega, k_{y}\right)=e^{i k_{x} \ell}$. The corresponding eigenvalue problem to solve is

$$
M_{1}\left(\begin{array}{l}
A^{+} \\
A^{-}
\end{array}\right)=\mu M_{2}\left(\begin{array}{l}
A^{+} \\
A^{-}
\end{array}\right) .
$$

Eventually, it allows us to obtain the Floquet-Bloquet wave number $k_{x} l$ in the first Brillouin zone $(-\pi, \pi)$ as a function of $\omega$ and $k_{y} \ell$.
[1] X. Hu and C. T. Chan, Phys. Rev. Lett. 95, 154501 (2005).

[2] C. Zhang, C. T. Chan, and X. Hu, Sci. Rep. 4, 6979 (2014).

[3] T. Bobinski, A. Eddi, P. Petitjeans, A. Maurel, and V. Pagneux, Appl. Phys. Lett. 107, 014101 (2015).

[4] G. Dupont, O. Kimmoun, B. Molin, S. Guenneau, and S. Enoch, Phys. Rev. E 91, 023010 (2015).

[5] H. Chen, J. Yang, J. Zi, and C. T. Chan, Europhys. Lett. 85, 24004 (2009).

[6] C. P. Berraquero, A. Maurel, P. Petitjeans, and V. Pagneux, Phys. Rev. E 88, 051002 (2013).

[7] M. Farhat, S. Enoch, S. Guenneau, and A. B. Movchan, Phys. Rev. Lett. 101, 134501 (2008).

[8] R. Porter and J. N. Newman, J. Fluid Mech. 750, 124 (2014).

[9] J. N. Newman, Eur. J. Mech. B/Fluids 47, 145 (2014).

[10] J. Xu, X. Jiang, N. Fang, E. Georget, R. Abdeddaim, J. M. Geffrin, M. Fahrat, P. Sabouroux, S. Enoch, and S. Guenneau, Sci. Rep. 5, 10678 (2015).
[11] A. Zareei and M. R. Alam, J. Fluid Mech. 778, 273 (2015).

[12] G. Dupont, S. Guenneau, O. Kimmoun, B. Molin, and S. Enoch, J. Fluid Mech. 796, R1 (2016).

[13] R. Porter, in World Scientific Handbook of Metamaterials and Plasmonics, edited by R. V. Craster and S. Guenneau (World Scientific, Singapore, 2017), Vol. 2, p. 680.

[14] R. R. Rosales and G. C. Papanicolaou, Stud. Appl. Math. 68, 89 (1983).

[15] W. Craig, P. Guyenne, D. P. Nicholls, and C. Sulem, Proc. R. Soc. London A 461, 839 (2005).

[16] M. Cathala, Monatsh. Math. 179, 325 (2016).

[17] P. Cobelli, A. Maurel, V. Pagneux, and P. Petitjeans, Exp. Fluids 46, 1037 (2009).

[18] A. Maurel, P. Cobelli, V. Pagneux, and P. Petitjeans, Appl. Opt. 48, 380 (2009).

[19] F. Barra, A. Maurel, V. Pagneux, and J. Zuniga, Phys. Rev. E 81, 066210 (2010) 